(C) [2008] IEEE. Reprinted, with permission, from [H. T. Nguyen, N. Ghevondian and T. W. Jones, Detection of nocturnal hypoglycemic episodes (natural occurrence) in children with Type 1 diabetes using an optimal Bayesian neural network algorithm, Engineering in Medicine and Biology Society, 2008. EMBS 2008. 30th Annual International Conference of the IEEE, 20-25 Aug. 2008]. This material is posted here with permission of the IEEE. Such ermission of the IEEE does not in any way imply IEEE endorsement of any of the University of Technology, Sydney's products or services. Internal or personal use of this material is permitted. However, permission to reprint/republish this material for advertising or promotional purposes or for creating new collective works for resale or redistribution must be obtained from the IEEE by writing to pubs-permissions@ieee.org. By choosing to view this document, you agree to all provisions of the copyright laws protecting it 


\title{
Detection of Nocturnal Hypoglycemic Episodes (Natural Occurrence) in Children with Type 1 Diabetes using an Optimal Bayesian Neural Network Algorithm
}

\author{
H. T. Nguyen, Senior Member, IEEE, N. Ghevondian, and T. W. Jones
}

\begin{abstract}
Hypoglycemia or low blood glucose is unpleasant and can result in unconsciousness, seizures and even death. It is a common and seruious side effect of insulin therapy in patients with diabetes. HypoMon is a non-invasive monitor that measures some physiological parameters continuously to provide detection of hypoglycemic episodes in Type 1 diabetes mellitus patients (T1DM). Based on heart rate and corrected QT interval of the ECG signal, we have continued to develop Bayesian neural network detection algorithms to recognize the presence of hypoglycemic episodes. From a clinical study of 16 children with T1DM, associated with nocturnal hypoglycemic episodes (natural occurrence), their heart rates increased $(\mathbf{1 . 0 3 3} \pm 0.242$ vs. $1.082 \pm 0.298, P<0.06)$ and their corrected $Q T$ intervals increased significantly $(1.031 \pm 0.086$ vs. $1.060 \pm 0.084$, $P<0.001)$. The overall data were organized into a training set $(8$ patients) and a test set (another 8 patients) randomly selected. Using the optimal Bayesian neural network with 10 hidden nodes which was derived from the training set with the highest log evidence, the sensitivity (true positive) value for detection of hypoglycemia in the test set is $89.2 \%$.
\end{abstract}

\section{INTRODUCTION}

$\mathrm{R}$ ESULTS of the Diabetes Control and Complications Trial (DCCT) Research Group in 1993 [1] showed that intensive insulin therapy for a mean of six years (maintaining glycemic levels to a target $\mathrm{HbA}_{1 \mathrm{c}}$ level of 7\%) as opposed to conventional therapy (with resultant mean $\mathrm{HbA}_{1 \mathrm{c}}$ level of $9 \%$ ) significantly lowered the risk for retinopathy by $47 \%$, nephropathy by $54 \%$ and for neuropathy by $60 \%$ [1-2].

However, episodes of hypoglycemia, especially at night, were common among people treated for T1DM, largely because usual insulin preparations do not adequately mimic the normal patterns of endogenous insulin secretion [2]. In the DDCT, patients assigned to intensive therapy experienced a threefold increase incidence of severe hypoglycemic episodes over those receiving conventional therapy $[1,3]$. In that report, severe hypoglycemic episodes are defined as those in which the patient required assistance to treat the event and had documented blood glucose levels

Manuscript received April 6, 2008. This work was supported in part by Juvenile Diabetes Research Foundation International under Regular Research Grant 1-2005-1055.

H. T. Nguyen is with Faculty of Engineering, University of Technology, Sydney, Broadway, NSW 2007, Australia (phone: +612-9514-2451; fax: +61 29514 2868; e-mail: Hung.Nguyen@uts.edu.au).

N..Ghevondian is with AIMedics Pty Ltd, Eveleigh, NSW 1430, Australia (e-mail: nejhdeh@aimedics.com).

T. W. Jones is with Princess Margaret Hospital, Subiaco, Perth, WA 6008, Australia (e-mail: tim.jones@health.wa.gov.au). less than $50 \mathrm{mg} / \mathrm{dl}(2.8 \mathrm{mmol} / \mathrm{l})$. Thus hypoglycemia proved to be a limiting factor in achieving improved diabetes control.

Symptoms of hypoglycemia arise from the activation of the autonomous central nervous systems (autonomic symptoms) and from reduced cerebral glucose consumption (neuroglycopenic symptoms), some of the latter being potentially life threatening. Autonomic symptoms (e.g., tachycardia, palpitations, shakiness, sweating) are activated before neuroglycopenic symptoms (e.g., reduced concentration, blurred vision, dizziness). Autonomic symptoms may provide the initial indication of the presence of hypoglycemia and allow the patient to recognise and correct the ensuing episode [6].

Nocturnal hypoglycemia is particularly dangerous because sleep reduces and may obscure autonomic counterregulatory responses, so that an initially mild episode may become severe. The risk of severe hypoglycemia is high at night, with at least $50 \%$ of all severe episodes occurring during that time [7]. Deficient glucose counter-regulation may also lead to severe hypoglycemia even with modest insulin elevations.

Recently, we have developed an optimal Bayesian neural network algorithm for the detection of hypoglycaemic episodes in T1DM children using physiological parameters. Using the data from 25 children with T1DM volunteered for the 4-hour glucose clamp study to provide 28 sets of physiological responses, we have found that hypoglcyemic episodes in T1DM children can be detected non-invasively and continuously [8-9].

In this paper, we develop an optimal Bayesian neural network algorithm for the detection of natural occurrence of nocturnal hypoglycemic episodes in T1DM children using physiological parameters such as heart rate, corrected QT interval and skin impedance. Section II provides an overview of the method used for non-invasive and continuous detection of hypoglycemia. Section III presents the development and results of an optimal Bayesian neural network used for the identification of nocturnal hypoglycemic episodes in T1DM children. Section IV provides a conclusion for this study.

\section{METHODS}

\section{A. Non-Invasive Hypoglycemia Monitor}

There is a limited number of non-invasive blood glucose monitoring systems currently available but each has specific drawbacks in terms of functioning, cost, reliability and obtrusiveness. Recently, GlucoWatch G2 Biographer from 
Cygnus Inc was designed to measure glucose levels up to 3 times per hour for 12 hours. The AutoSensor (the disposable component) which was attached to the back of the GlucoWatch monitor and adhered to the skin will provide 12 hours of measurement. The product used reverse iontophoresis to extract and measure glucose levels noninvasively using interstitial fluid. It had to be calibrated before each measurement period and required a two-hour warm-up period. It required costly disposable components, the gel pads must be replaced after each use, sweating might cause skipped readings, and the measurement had a time delay of about 10-15 minutes. As a result of these limitations this device is no longer available.

Intensive research has been devoted to the development of hypoglycaemia alarms, exploiting principles that range from detecting changes in skin conductance (due to sweating) to measurements, by glucose sensors, of subcutaneous tissue glucose concentrations (Pickup, 2000; Rebrin; 1999). However, none of these have proved sufficiently reliable or unobtrusive.

Although real-time continuous glucose monitoring systems (CGMS) are now available to give real-time estimations of glucose levels, these still lack the sensitivity to be used as alarms. For the MiniMed Medtronic (Northridge, CA) CGMS, the median error was reported as $10-15 \%$ at a plasma glucose of 4-10 mmol/1 [10-12] and the low efficacy of CGMS (79.1\% sensitivity) in detecting unrecognised hypoglycaemia has been confirmed [13]. For the Abbott Freestyle Navigator CGMS, the sensor accuracy was lowest during hypoglycaemia $(3.9 \mathrm{mmol} / \mathrm{l})$, with the median absolute relative difference (ARD) reported as $26.4 \%$ [14]. As these are median values, the errors may be significantly greater and, as a result, the manufacturers do not recommend their use as an alarm.

We have developed a continuous non-invasive hypoglycemia monitor which uses physiological responses [8-9]. HypoMon ${ }^{\circledR}$ (Hypoglycemia Monitor) from AIMedics Pty Ltd (Fig. 1) is a non-invasive monitor that measures physiological parameters continuously to provide detection of hypoglycemic episodes in Type 1 diabetes mellitus patients (T1DM). The system consists of a battery-powered chest belt worn that houses a set of four skin-surface biosensor electrodes for the measurement of physiological parameters and a hand-held receiver computer.

The sensors are composed of a conductive polymer-based material such as polypyrrole which has low impedance and low noise characteristics. These characteristics enable the sensors to measure both skin impedance and quality ECG signals. The chest belt digitizes, encrypts and transmits the measured parameters to the receiver computer which analyzes and identifies hypoglycemic episodes. An alarm system is available for warning various stages of hypoglycemia.

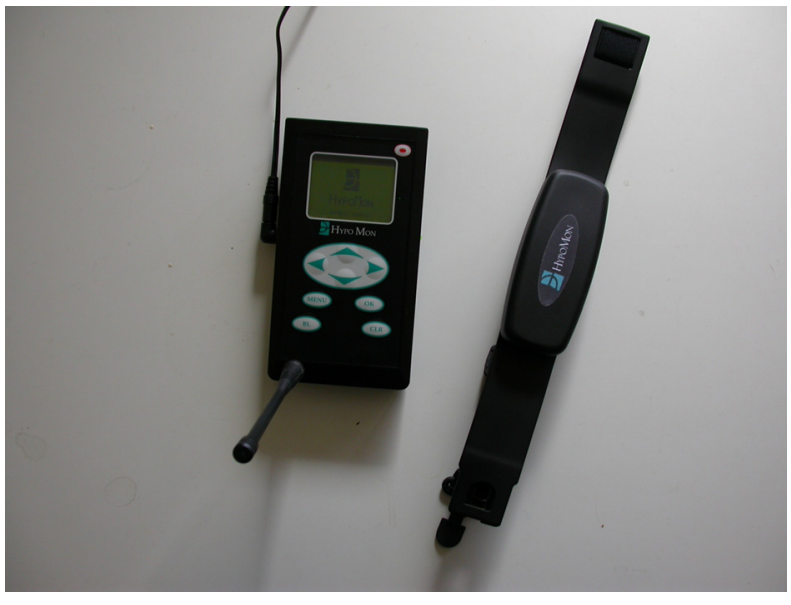

Fig. 1. HypoMon (AIMedics Pty Ltd)

\section{B. Bayesian Neural Network}

Bayesian neural networks were firstly introduced by MacKay as a practical and powerful means to improve the generalisation of neural networks [16-19]. Bayesian learning of multi-layer perceptron neural networks is performed by considering Gaussian probability distributions of the weights which can give the best generalisation [16-17]. In particular, the weights $w$ in network $X$ are adjusted to their most probable values given the training data $D$. Specifically, the posterior distribution of the weights can be computed using Bayes' rule as follows

$$
p(w \mid D, X)=\frac{p(D \mid w, X) p(w \mid X)}{p(D \mid X)}
$$

where $p(D \mid w, X)$ is the likelihood function, which contains information about the weights from observations and the prior distribution $p(w \mid X)$ contains information about the weights from background knowledge. The denominator, $p(D \mid X)$, is known as the evidence for network $X$.

Regularisation can be used to prevent any weights becoming excessively large, which can lead to poor generalisation. For a multi-layer perceptron neural network classifier with $G$ groups of weights and biases, a weight decay penalty term proportional to the sum of squares of the weights and biases is added to the data error function $E_{D}$ to obtain the cost function

$$
S=E_{D}+\sum_{g=1}^{G} \xi_{g} E_{W_{g}}, E_{W_{g}}=\frac{1}{2}\left\|w_{g}\right\|^{2} \quad(g=1, \ldots, G)
$$

where $S$ is called the cost function, $\xi_{g}$ is a non-negative scalar, sometimes knows as a hyperparameter, ensuring the distribution of weights and biases in group $g$ and $w_{g}$ is the vector of weights and biases in group $g$. 
In network training, the hyperparameters are initialised to be arbitrary small values. The cost function is then minimised using an advanced optimisation technique. When the cost function has reached a local minimum, the hyperparameter $\xi_{g}(g=1, \ldots, G)$ must be re-estimated. This task requires computing the Hessian matrix of the cost function:

$$
A=H+\sum_{g=1}^{G} \xi_{g} I_{g}
$$

where $H$ is the Hessian matrix of $E_{D}$ and $I_{g}$ is the identity matrix, which selects weights in the $g$ th group. The number of 'well-determined' weights $\gamma_{g}$ in group $g$ is calculated based on the old value of $\xi_{g}$ as follows [15]

$$
\gamma_{g}=W_{g}-\xi_{g} \operatorname{tr}\left(A^{-1} I_{g}\right), \xi_{g}=\frac{\gamma_{g}}{2 E_{W_{g}}} \quad(g=1, \ldots, G)
$$

The hyperparameters need to be re-estimated several times until the cost function value ceases to change significantly between consecutive re-estimation periods. After the network training is completed, the values of parameters $\gamma_{g}$ and $\xi_{g}$ are then used to compute the $\log$ evidence of network $X_{i}$ having $M$ hidden nodes as follows [18-19]

$$
\begin{aligned}
\ln E v\left(X_{i}\right) & =-S+\sum_{g=1}^{G} \frac{W_{g}}{2} \ln \xi_{g}-\frac{1}{2} \ln |A|+\ln M !+M \ln 2 \\
& +\sum_{g=1}^{G} \frac{1}{2}\left(\frac{4 \pi}{\gamma_{g}}\right)-G \ln (\ln \Omega)
\end{aligned}
$$

where $W_{g}$ is the number of weights and biases in group $g$, and $\Omega$ is set to be $10^{3}$ [19]. However, $\Omega$ is a minor factor because it is the same for all models and therefore does not effect to the relative comparison of log evidence of different network architectures. The above Equation is used to compare different networks having different numbers of hidden nodes. The best network will be selected with the highest log evidence.

\section{Study}

Sixteen children with T1DM volunteered for the 10-hour overnight hypoglycemia study at the Princess Margaret Hospital for Children in Perth, Australia. Each patient was monitored overnight for the natural occurrence of nocturnal hypoglycemia. Data were collected with approval from Women's and Children's Health Service, Department of Health, Government of Western Australia, and with informed consent.

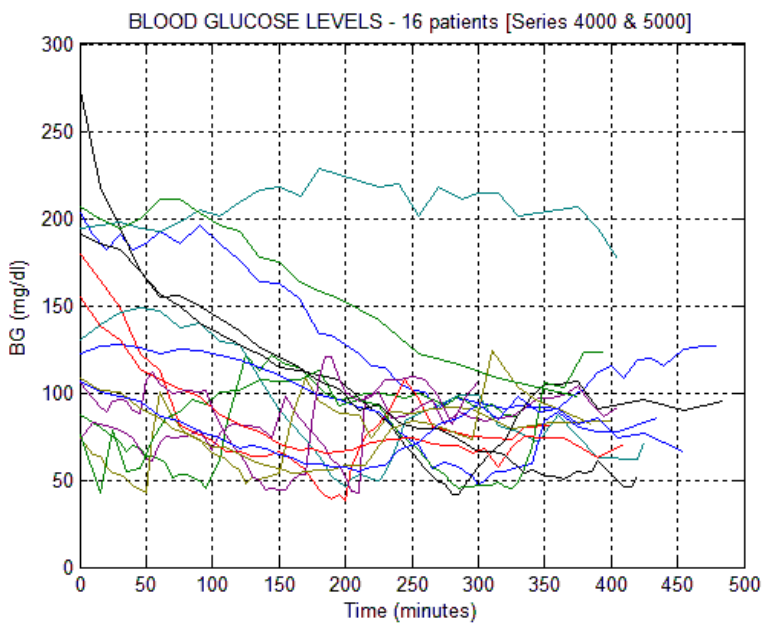

Fig. 2. Actual blood glucose level profiles in 16 T1DM children

HypoMon was used to measure the required physiological parameters, while the actual blood glucose (BG) levels were collected as reference using Yellow Spring Instruments. The main parameters used for the detection of hypoglycemia are the heart rate, corrected QT interval and skin impedance. The actual blood glucose profiles for 16 T1DM children are shown in Fig. 2.

\section{RESULTS}

HypoMon was used to measure the relevant physiological responses. The four skin-surface bio-sensor electrodes are multiplexed and shared to measure both skin impedance and ECG signals.

The responses from 16 T1DM children exhibit significant changes during the hypoglycemia phase against the non-hypoglycemia phase. Normalization was used to reduce patient-to-patient variability and to enable group comparison by dividing the patient's heart rate, corrected QT interval and skin impedance by his/her corresponding values at time zero. The study shows that associated with hypoglycemic episodes in 16 T1DM children, using normalized values, their heart rates increase $(1.082 \pm 0.298$ vs. $1.033 \pm 0.242, \mathrm{P}<0.06)$ and their corrected QT intervals increase significantly $(1.060 \pm 0.084$ vs. $1.031 \pm 0.086$, $\mathrm{P}<0.001$ ). However, in this clinical study (natural occurrence), the reduction of skin impedances of the patients were not strong $(1.111 \pm 1.460$ vs. $1.108 \pm 1.277$, $\mathrm{P}<0.984$ ). It should noted that in our previous 4-hour glucose clamp study [8-9], skin impedances reduced significantly during hypoglycemic episodes.

The detection of hypoglycemic episodes $(\mathrm{BG}<=60$ $\mathrm{mg} / \mathrm{dl}$ ) using these variables is based on an optimal Bayesian neural network algorithm developed from the obtained clinical data. This neural network has a multilayer feed-forward neural network structure with one hidden node layer and one output node layer. In effect, it estimates the presence of hypoglycemia at sampling period $\mathrm{k}$ based 
on the basis of the data at sampling period $\mathrm{k}$ and the previous data at sampling period $\mathrm{k}-1$. In general, the sampling period is 5 minutes and approximately 30 data points are used for each patient.

The overall data set consisted of a training set and a test set, each with 8 cases randomly selected. For these, the whole data set which included both hypoglycemia data part and non-hypoglycemia data part were used. For optimal robustness of the evaluation, we applied the evidence framework for Bayesian inference to the training set and found the feed forward neural network architecture with 10 hidden nodes yielded the highest evidence as shown in Fig. 3. The final feed-forward multi-layer neural network had heart rate, corrected QT interval and skin impedance as inputs, 10 hidden nodes and 1 output node (estimated blood glucose level). From the optimal neural network which was derived from the training set with the highest log evidence, the sensitivity (true positive) value for detection of hypoglycemia in the test set is $89.2 \%$.

\section{CONCLUSION}

The above result indicates that hypoglycemic episodes in T1DM children can be detected non-invasively and continuously effectively from the real-time physiological responses measured by HypoMon. It is noted that the relevant physiological parameters (QTc interval, heart rate and skin impedance) are less correlated to the actual blood glucose levels when the patients experienced hypoglycemic episodes through natural occurrence against the situation when clamp studies were performed. In this study, the sensitivity obtained by the hypoglycemia detection neural network is still low and its overall accuracy could be improved. A more advanced neural network algorithm will be developed to improve its overall accuracy further.

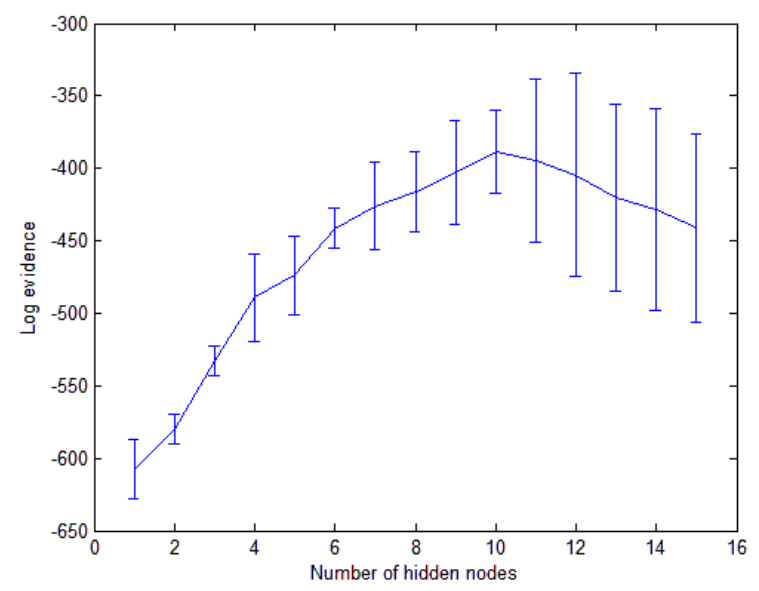

Fig. 3. Evidence framework for Bayesian inference

\section{REFERENCES}

[1] DCCT Research Group (1993): The effect of intensive treatment of diabetes on the development and progression of long-term complications in IDDM. N. Eng. J. Med., 329, 977-986.

[2] Yale J-F (2004). Nocturnal hypoglycemia in patients with insulintreated diabetes. Diab Research and Clinical Practice, 65S:S41S46.

[3] DCCT Research Group (1995): Adverse events and their association with treatment regimens in the Diabetes Control and Complications Trial. Diabetes Care, 18, 1415-1427.

[4] Cryer PE (2002): Hypoglycaemia: the limiting factor in the glycaemic management of type I and type II diabetes. Diabetologia, 45, 937-948.

[5] Davis S, Alonso MD (2004): Hypoglycemia as a barrier to glycemic control. J. Of Diabetes and its Complications, 18, 60-68.

[6] Clarke WL, Cox DJ, Gonder-Frederick LA, Julian D, Schlundt D, Polonsky W (1995): Reduced awareness of hypoglycemia in adults with IDDM. A prospective study of hypoglycemic frequency and associated symptoms. Diabetes Care; 18:517-522.

[7] DCCT Research Group (1991): Epidemiology of severe hypoglycemia in the diabetes control and complication trial. Am. J. Med., 90, 450-459.

[8] Nguyen HT, Ghevondian N, Nguyen ST, Jones T (2007): Detection of Hypoglycemic Episodes in Children with Type 1 Diabetes using an Optimal Bayesian Neural Network Algorithm, $29^{\text {th }}$ Annual International Conference of the IEEE Engineering in Medicine and Biology Society, Lyon, France, 3140-3143.

[9] Nguyen HT, Ghevondian N, Jones T (2006): Neural-network Detection of Hypoglycemic Episodes in Children with Type 1 Diabetes using Physiological Parameters, $28^{\text {th }}$ Annual Int Conf of the IEEE Eng in Med and Biol Society, New York, 6053-6056.

[10] Directnet Study Group (2005). Accuracy of the modified CGMS sensor in an outpatient setting. Diabetes Technol Ther 7:109-114.

[11] Directnet Study Group (2006). Evaluation of factors affecting CGMS calibration. Diabetes Technol Ther 8:318-325.

[12] The Diabetes Research in Children Network Study Group (2004). The accuracy of the freestyle navigator CGMS in children with Type 1 diabetes. Diabetes Care 27:734-738.

[13] Maia FFR, Araujo LR (2007). Efficacy of continous glucose monitoring system (CGMS) to detect postprandial hyperglycemia and unrecognized hypoglycemia in type 1 diabetic patients. Diabetes Research and Clinical Practice 75:30-34.

[14] Weinstein RL et al Accuracy of the freestyle navigator CGMS: comparison with frequent laboratory measurements. Diabetes Care 30:1125-1130, 2007

[15] Gale EAM, Bennett T, MacDonald IA, Holst JJ, Matthews JA (1983): The physiological effects of insulin-induced hypoglycemia in man: responses at differing levels of blood glucose, Clin. Sciences, 65, 263-271.

[16] Heller SR, Macdonald IA (1991): Physiological disturbances in hypoglycemia: effect on subjective awareness. Clin. Sci., 81, 1-9.

[17] Harris ND, Baykouchev SB, Marques JL (1996): A portable system for monitoring physiological responses to hypoglycemia. $J$. Med.Eng. \& Tech., 20, 196-202.

[18] Tattersall RB, Gill GV (1991): Unexplained death of type 1 diabetic patients. Diabetic Med; 8:49-58.

[15] Marques JL, George E, et al. (1997): Altered ventricular repolarisation during hypoglycaemic in patient with diabetes. Diabetic Med; 8:648-654.

[19] MacKay D (1992): The evidence framework applied to classification networks. Neural Computation; 4:720-736.

[20] MacKay D (1992): A practical Bayesian framework for backpropagation networks. Comp. \& Neural Systems; 4:448-472.

[21] Penny WD, Roberts SJ (1999): Bayesian neural networks for classification: how useful is the evidence framework. Neural Networks; 12:877-892.

[22] Thodberg HH (1996): A review of Bayesian neural networks with an application to near infrared spectroscopy. IEEE Transactions on Neural Networks; 7:56-72. 